\title{
Insight into Kansei Color Combinations in Interactive User Interface Designing
}

\author{
K.G.D. Tharangie ${ }^{1}$, Shuichi Matsuzaki ${ }^{2}$, Ashu Marasinghe ${ }^{1}$, and Koichi Yamada ${ }^{1}$ \\ ${ }^{1}$ Department of Informatoion Science and Control Engineering \\ ${ }^{2}$ Department of Electrical Engineering \\ Nagaoka University of Technology, Japan \\ tharangie@yahoo.com, shmatsu@vos.nagaokaut.ac.jp, \\ \{ashu, yamada\} @kjs.nagaokaut.ac.jp
}

\begin{abstract}
Color has a major impact on Human Computer Interaction. Although there is a very thin line between appropriate and inappropriate use of color, if used properly, color can be a powerful tool to improve the usefulness of an interactive interface in a wide variety of areas. On the contrary the excessive or inappropriate use of color can severely hinder the functionality and usability of an interface accordingly. A good visual design provides higher level of user satisfaction and further aids with conveying the intended message to its audience. In this paper we focus on one requisite aspect of visual design as such the Color, revealing one hidden dimension of color; Affectivity, by acquiring prospective user's concealed color aesthetic preferences, employing Kansei Engineering Assessing System with respect to interactive Interfaces.
\end{abstract}

Keywords: Interactive environment, Kansei Engineering, color, Visual design, Affective color.

\section{Introduction}

There is an ample of research work already carried out in the field of color aesthetics and interactive design. There is abundance of design guidelines and recommendations available for visual design including the use of colors in interface designing, data visualization, Interactive interfaces designing etc; nevertheless these guidelines conflict with one another as there are no precise protocols for interface designing. Therefore, designers resort to follow an experimental approach, which also becomes the guideline for him with his level of maturity.

In this research, we neither associate colors with any already existing color associations nor consider the color psychology, color symbolism or color theories; instead, we are employing Kansei Engineering to perform this task for non designer and also for designers. We collect a number of adjectives that people use to express their feelings about the objects that they find in their daily life and combine them with the trait words in the domain. In other words, request prospective users or experimental subjects to select the adjectives that which they think is useful to describe the characteristics of colors. Furthermore these adjective elaborate the inner thoughts of the user, 
and can be used as a measurement to decide the relevance or usability of color in a particular situation. In brief, the main purpose of this research is to listen to what user has to say about the use of colors in already existing design materials, and by analyzing, organizing, modeling and presenting them as a pool of knowledge in a certain way as solutions for designers to design a better Interactive environments for the respective users.

Distinct users from different cultural, educational, professional backgrounds may have different kinds of individual favours. That is the very reason why designers cannot accommodate already existing designing protocols as it is, when designing for a specific user group. In this research we attempt to explore user's hidden point of views in a form of affective parameters, and analyze that to generalise and derive common human affective patterns which everyone share as human beings. It is not necessary that red should represent anger, as described in color psychology. The prime focus of the research is that, how people feel about the red when it is associated with other colors, represented as background color, foreground color. Is it easy to read? Does it really contribute some value to usability of the Interactive learning environment? Does it increase the visual search? or does it make the interactivity more exciting and challenging? What colors we should use to emphasise, highlight important points? Concisely, using Kansei Engineering we capture the affective parameters from the actual users, and process it in Kansei Engineering to output better solutions to design a products and services.

\section{What Is Kansei?}

Several Scholars has given various definitions for Kansei. What is highlight by many of them is that Kansei is a subjective internal process in the brain, which activated by an external stimuli (see Fig. 1). Nagamachi further explains this concept by articulating that Kansei is an individual's subjective impression from a certain artefact, environment, or situation using all the senses of sight, hearing, feeling, smell, taste as well as recognition"[1]. Lee et al. further elaborating the word 'Kansei' says that the word Kansei is embedded with more semantics such as sensibility, sense, sensitivity, aesthetics, emotion, affection and intuition. [2]

\subsection{Kansei Engineering}

In a very special manner via human affect, feelings, emotions Kansei Engineering is able to capture the information which we sense from five sensory organs as an input and send though various screening levels to produce the final output. The Engineering process (see fig. 2) is a network which is interconnected and it recognises the correct functions to generate inter-relationships and produce the output with sense. Before using Kansei as an engineering method, there are three focal points one should understand about Kansei; Firstly How to understand Kansei, secondly how to reflect and translate Kansei, finally how to create system and organization for Kansei Oriented Design. Even though it is very difficult to capture one's Kansei; it can be approximately measured using fallowing four different methods [1] People's behaviours and actions, Words (spoken), Facial and body expressions, Physiological responses (Heart rate, EMG, EEG). 


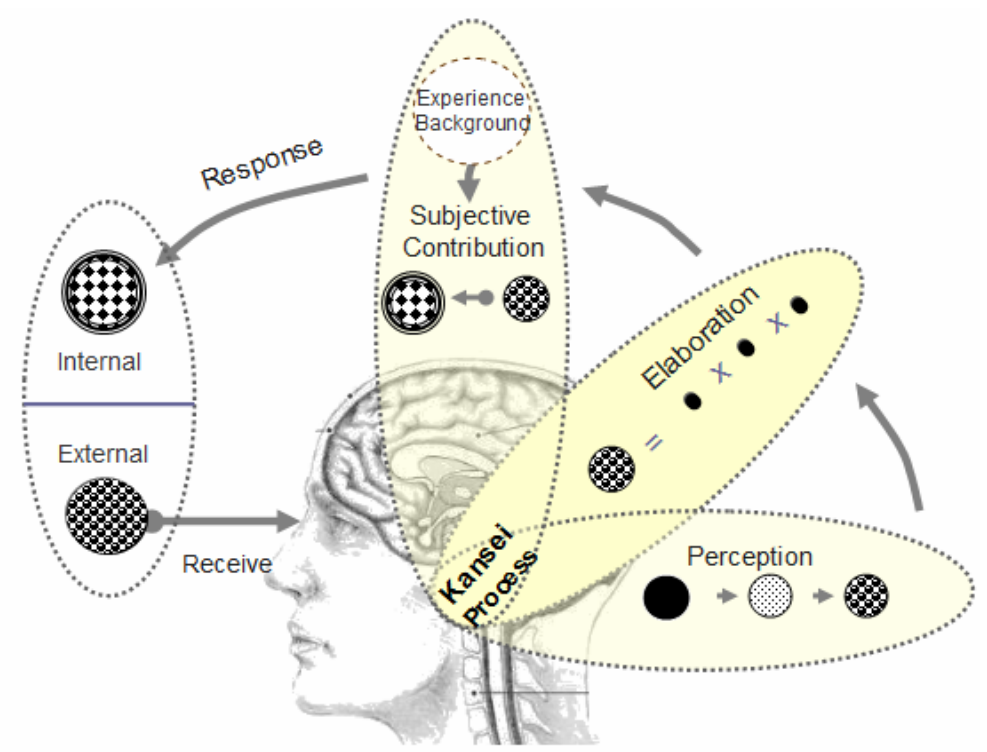

Fig. 1. Kansei is an interpretation of a subjective Experience. A person perceives an object at first and further elaborates it to get a better understanding of the object. During this process of conceptualization persons' experience background influence predominantly in generating his subjective impression/ Kansei; for generating the internal picture.

Above mentioned five measurement methods again can be categorized under two main categories such as psychological and physiological measurements. Some Kansei engineers prefer Physiological methods where they can directly measure user's facial expressions, Heart rate, EMG, EEG etc. during a user is actively interacting with the experiment. However some engineers believe Physiological measurements are not exactly Kansei itself rather a by-product of Kansei. Therefore engineers commonly accommodate more elaborative semantic differential methods. In semantic differential method, semantic scales are prepared using emotional related adjectives combined with trait words in the domain. In addition to Semantic Differential methods (SD methods), engineer can also get feedback from subjects' comments and recommendations; a qualitative measurement method, which expresses user preferences in their own words as answers for an open ended questionnaires.

In semantic differential method, engineer collect Kansei words or adjectives related to the product domain (in this research emotion related adjectives which express the attributes of colors in respect to interactive interfaces), and categorize adjectives that represent the user preferences and opinions in the particular domain and test them against the 5 or 7 point (Semantic Differential SD) scale [3]. After gathering SD data and analyzing statistically, Engineer makes suggestions for the designer to amend his design procedure with improved sense. If gathered result data was represent by a pyramid, primary data belongs in the bottom level or the basement of the pyramid. When data was analysed further, according to its refined level, it goes higher in the pyramid until it reach the peak of the pyramid where we find the highest level of Kansei or general Kansei. Lower degree of Kansei is more subjective or individual than 
higher levels of Kansei. When Engineer analysed and refined the data it contributes to build up higher levels of Kansei. Highest level of Kansei is more refined and generalised data of subjective Kansei and from that data new design principles derives.

Although the initial aims of Kansei Engineering was to build-up a methodology that is capable of constructing industrial products and services which reflect the users' personal preferences and requirements; currently it is emerging into a new direction, where it can assist with fundamental technical issues in Human Computer Interaction for realising safety and pleasantness of individuals, which is considered to be the most fundamental problem in the coming information network society. [6]

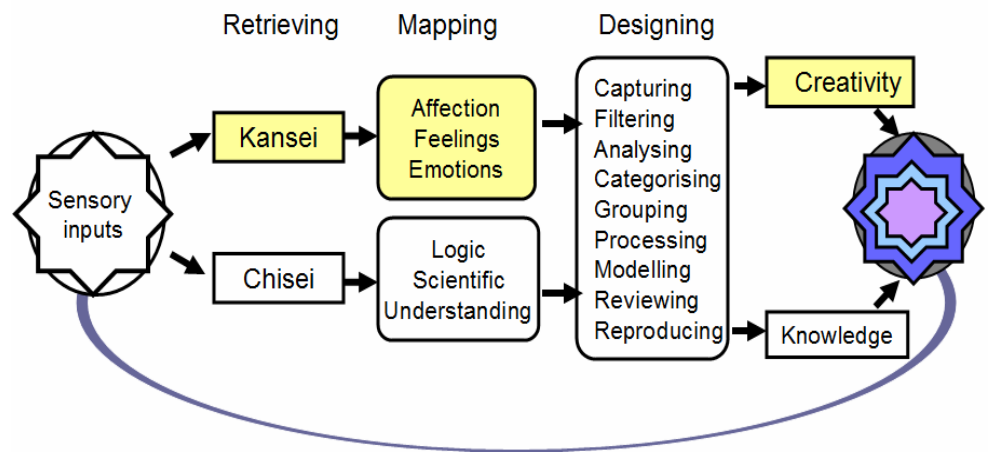

Fig. 2. Kansei Processing System

Fig. 2 represents Kansei Engineering process in cooperation with Chisei. In order to explain the domain of Kansei clearly, Lee et al., [2] gained the aid from the Japanese word Chisei. While Kansei is closely connected with affective emotional values, Chisei is closely connected with logic recognition and understanding. Both of these concepts are activated by sensory inputs but going through two different ways of mapping and processing and finally out put the result as creativity and knowledge.

\section{Method}

Subjects are 20 male and female under graduate University students in Information Technology Engineering in Sri Lanka. They are between the age of 20 and 30. No subjects reported in color deficiency. All of the subjects reported extensive experience with computers and most of them used the computer for more than 3 years during the period of their study, thoroughly experienced in programming and computer software handling. None of them were specially trained for any specific color related applications. Moreover, the research was conducted in paper medium in daylight and normal classroom condition.

Research methodology consists of three consecutive sessions: Preliminary data acquisition (collecting Kansei words and collecting computer interfaces), detailed data gathering questionnaire, testing with bipolar scales. Eleven basic colors used in computer interface designing; known universally, as the colors that "almost never confused" (red, pink, purple, blue, green, yellow, orange, brown, white, grey, black) accommodated as the basic testing color palette (see table 1). 
Table 1. Selected Color Palette

\begin{tabular}{|c|c|}
\hline Colour & RGB Value \\
\hline Red & $255,0,0$ \\
\hline Orange & $255,165,0$ \\
\hline Brown & $165,42,42$ \\
\hline Yellow & $255,255,0$ \\
\hline Green & $0,128,0$ \\
\hline Blue & $0,0,255$ \\
\hline Purple & $128,0,128$, \\
\hline Grey & $128,128,128$ \\
\hline Pink & $255,192,203$ \\
\hline White & $255,255,255$ \\
\hline Black & $0,0,0$ \\
\hline
\end{tabular}

Research was conducted in the morning in an enclosed quiet room in normal day light conditions. Questionnaires were conducted in subjects' native language (Sinhala). As mentioned above research questionnaire was designed employing user comments; a qualitative measuring methods and semantic differential scale quantitative measuring methods. Employing above two methods the Kansei Color questionnaire was prepared to address the following areas. Kansei color questionnaire employed for this research consists of two main sections. First section is the open-ended questionnaire. Second section consists of color testing with semantic differential scales. Experiment was conducted in three consecutive stages as fallows; Preliminary data acquisition, Detailed data gathering questionnaire, Testing against bipolar scales. Varieties of questions in questionnaire are as fallows.

- Selection of favourite colors and reason for selecting these colors.

- Matching colors and Kansei words

- $\quad$ Suggesting Kansei words for respective colors

- Color interface; testing background, font and theme colors

- Difference between less colors interfaces and Colorful interfaces

- $\quad$ Testing colors with Semantic Differential scales

\section{Result and Discussion}

\subsection{Affective Color}

Fig. 3 is an example for the capability of Kansei Engineering on acquiring people's hidden aesthetic preferences and quantifying those using semantic measurements of Kansei words. Furthermore, these graphs summarise the color preferences of the test subjects, for four of the Kansei words chosen in the study such as Pleasant, Boring, Exciting and Disgusted. It comprehends except colors brown, grey, black and red, other colors were considered as comparatively pleasant color choices.

When designing Interactive Interfaces, designers has to make preferable color choices or select preferable color combinations. Kansei Engineering can address that 
problem employing Kansei words. Using Kansei words, Kansei Engineer can measure numerous user preferences about color combinations and analyze them statistically to find a general or abstract pattern. Examining this result designer can get a basic idea about what color choices to make, what colours can be grouped together, and what colours are more compatible with chosen colors. In this research, for the Kansei word Boring and Disgusted subjects have again eminently selected black, brown and grey, colors and comparatively eliminated the others. Furthermore result of Kansei word Pleasant; blue, green, yellow, orange etc. have been selected as not boring and disgusted colors. The result of the Kansei word Exciting also assert the results of first Kansei word pleasant and illustrates that yellow, blue, green, pink and orange are preferable color choices for an interactive environment.
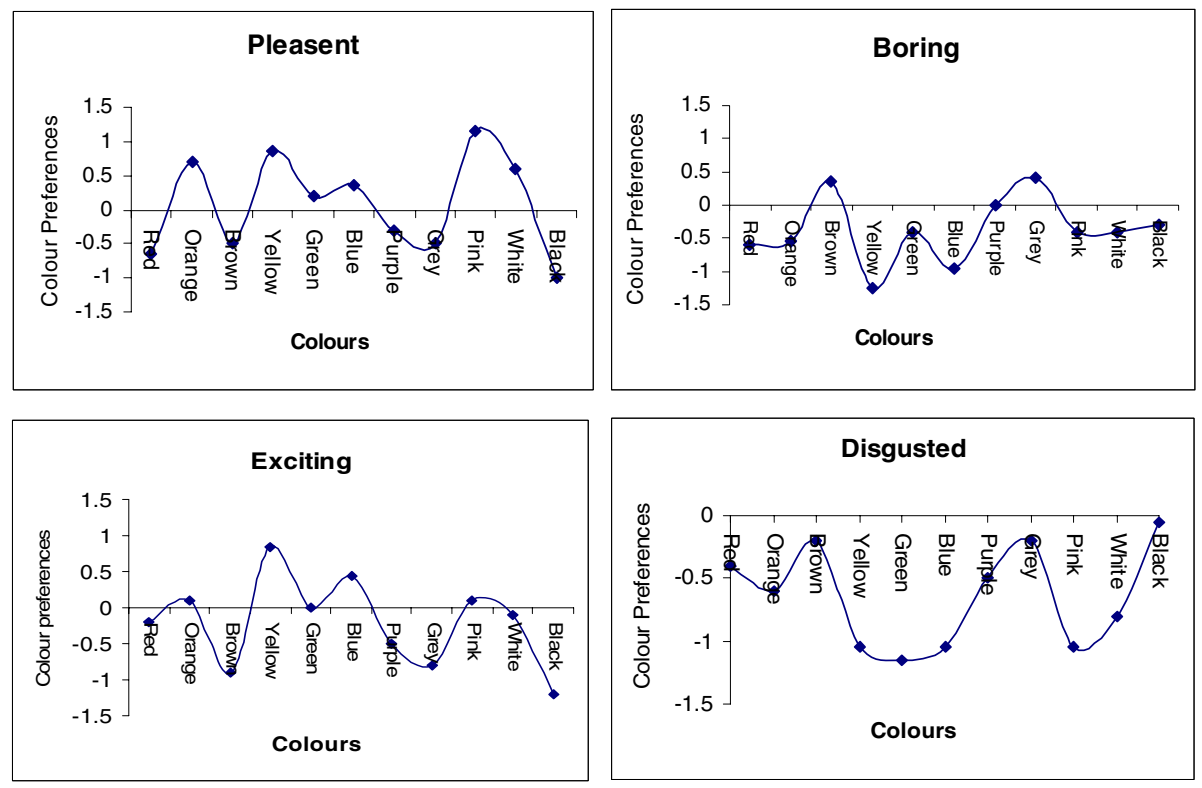

Fig. 3. Variation of color for Kansei words Pleasant, Boring, Exciting and Disgusted

Above mentioned result does not convey that each and every time when designer intend to design a user interface he needs to conduct a Kansei research. There are already existing Kansei expert systems, which particularly non designers but also designers can employ when selecting color combinations or designer himself can conduct a simple Kansei research for prospective users and grasp the color choices of the user through his affective/Kansei expressions. Nevertheless, the important point that should be noted here is not Kansei Engineering itself but apart from the all color physics relating to colour, there is another aspect or dimension of colors, which influence the user considerably but we apparently ignore as designers.

Moreover, results reveal that as colors, black, grey and brown are not so exciting or pleasant while colors as blue, yellow, pink green comparatively have higher 
preferences. With regard to this results, when designer select so called boring, unpleasant colors, they have to be careful about to what extend they can use those colors or how they can balance these colors to make color combinations to be combined with highly preferred colors. As per the results, preferences for the theme color were chosen in descending order; blue, green, white, yellow, pink etc as such. This result also confirms that colors that preferred as pleasant and exciting colors (see fig. 3) are also good color choices for theme colors.

However, this selection of vivid colours does not mean that black, grey and brown are unusable colors. Although they are not so preferred as the other colors, a good designer can manipulate these colors blending in right proportions in color combinations to highlight the other colors or balance or neutral the contrast of eminent colors. If one makes a crucial color choice and if he is not so certain about that colour combination, and if many other design matters depend on this choice, simple semantic differential analysis test with Kansei words will be immensely assistive.

\subsection{Color vs. Black and White}

Result reveals that $85 \%$ of the subjects preferred having colored interactive interfaces than black and white or mono color interfaces. If one examined the above graph (see fig. 4) it would be easy to realise that color environments are preferred over black and white; vivid colors were given higher ranking in positive Kansei words region than black and white. Nevertheless, it should be noted that; although black is not a popular color in this research questionnaire results, white was preferred more than some eminent colors such as blue and yellow. This research was conducted in Sri Lanka. In Sri Lankan culture black is considered to be the 'colour of Evil' and white is meant to be the 'colour of Sadness'. Another aspect of the same is that the black is regarded being the 'colour of corruption' and white is reckoned as the 'colour of purity'. These are cultural codes that a person learns through his socialization. Therefore, it is imperative to remember that culture codes play a major role in the process of generating 'subjective impressions' in one's mind. Solution for this problem is that the culture specific Kansei engineering and finally generalisation with comparison.
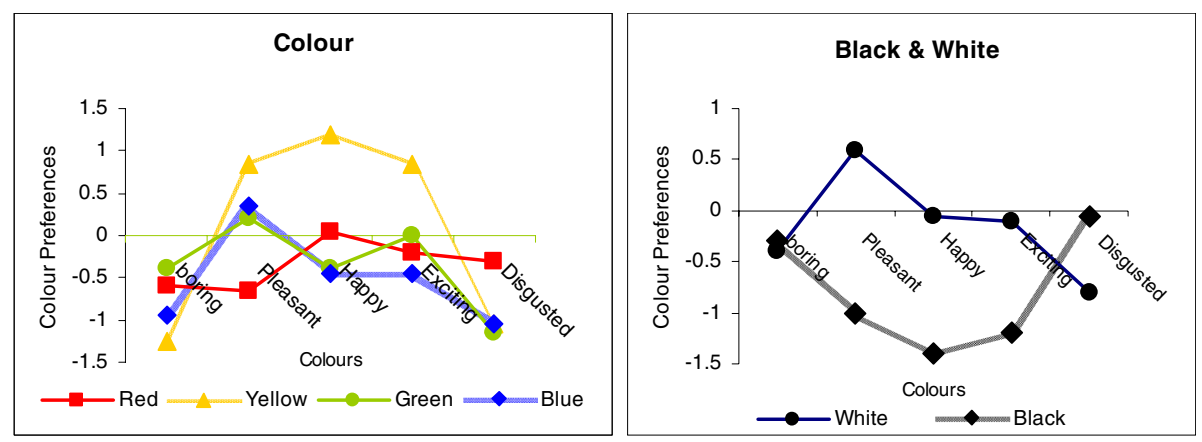

Fig. 4. Variation of color for Kansei words for color Vs Black \& White 


\subsection{Color Temperature and Affect}

Almost all the subjects considered good functionality is more important than good visual presentation or an attractive visual environment, but color add excitement and flavour to the learning activity. (Warm colors that generate warm feelings, so as they draw attention instantly than others which put our mind at agitation; uncomfortable to associate for a longer period of time to fig. 5) Warm colors such as red, yellow, pink and Orange can be utilised to draw attention to particular areas of the Interface and also in assistance to enhance the visual search, thus when someone decides give away a larger area of their interface to a warm colour, it should be done with caution. According to their affective nature warm colors fatigue the eye as well as the mind. On the contrary, Cool colors are pleasing to the eye as well as to the mind which make them good candidates for background colors and theme colors. However one should keep in mind that we can not talk about colors in terms of color Hue alone, the other attributes of the colors such as brightness and saturation also contributes to the color temperature, accordingly the affective quality of colors. [4], [5]

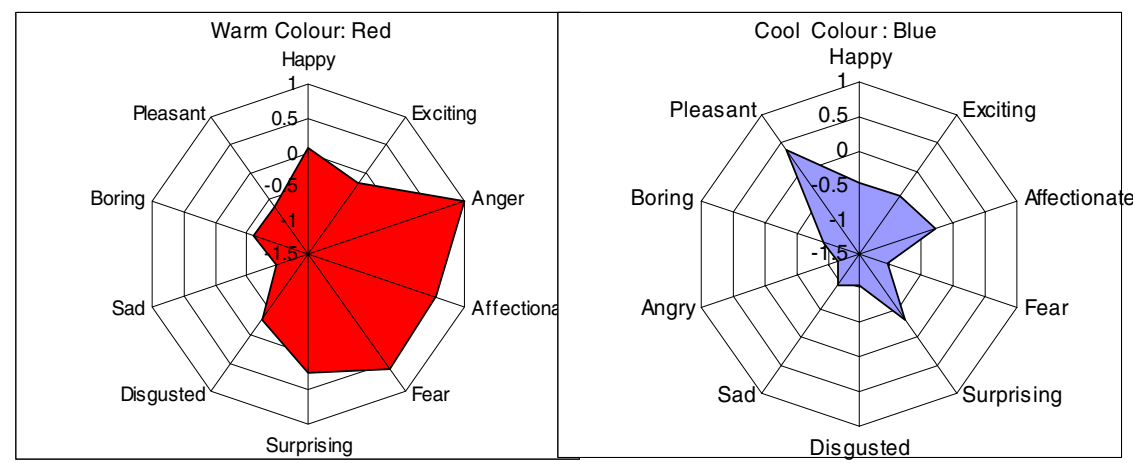

Fig. 5. Warm colors intense towards warm feelings and cool colors intense towards cool feelings

\subsection{Color Relations}

Relationship that each Kansei words has with colour, works as a grouping mechanism for Kansei words. Ten Kansei words, which were chosen out from more than 500 adjectives as the result of highest ranking by the subjects, were reduced to 4 factors or groups adopting factor analysis. i.e. Intense, Dull, Lively and Calm. For an instance, the factor Lively, consist of Kansei words such as happy, exciting and pleasant. Kansei words in one factor, strongly correlated to each other and significant at $95 \%$ level of confidence. The important point to make here is that, colors that work prominently behind the screen to form these Kansei words groups can be mapped and grouped under the above mentioned factors. Colour ranking for Kansei words were done using non parametric statistical method chi-square. By analyzing the ranking result of colors, engineer can derive what is the most suitable colour to represent respective Kansei words. Fig. 6 illustrates the fluctuations of variability of factors according to 
colours. This graph approximately illustrates the representation of respective colours in each factor.

Following is the summary of the section of Interface design related questions. Output of these results is also aligning with the result of quantitative questionnaire in the above sections. Appropriate using of colors provides a pleasing comfortable environment to work with. Color can assist with creating better mental models i.e. creating a hierarchy in the interactive environment, by assisting the user to understand the structure of the system. Consistency of color makes a virtual structure and supports the navigation. Pleasant colors make the interactive environment a pleasing place to work and it reduces boredom and sway in the readers mind towards enthusiasm. Using different shades and tints in the design, good designer can create elegant aesthetic pattern, which make the interface a special and comfortable place to interact with. On the contrary, results reveal that wrong use of color has an ability to make the environment more complex and noisy and lead to miscommunication.

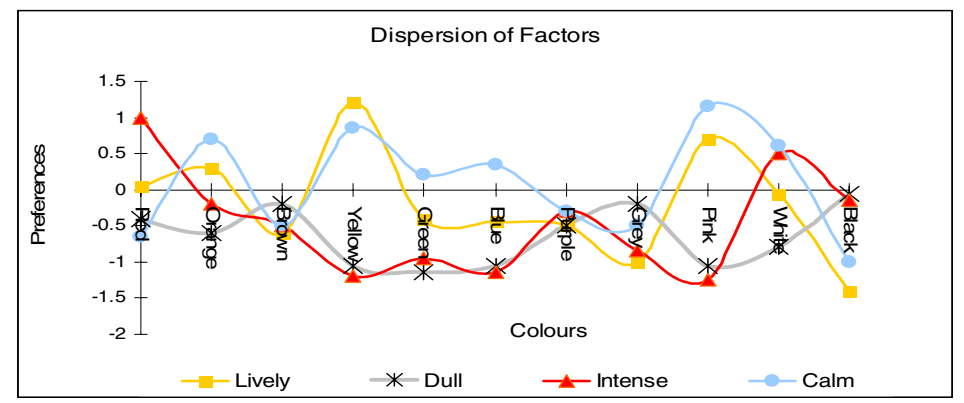

Fig. 6. Dispersion of 4 derived factors Calm, Lively, Intense and Dull

With all the color knowledge, simultaneously, it is an added advantage to have common understanding of affective parameters for colors for proper communication and coordination of colour for interactive Interface Design. Unnecessary utilisation of colors in design may lead to unattractive interactive interfaces which user is not willing to communicate with. Finally it is very important to accommodate color deficiencies; color itself should not be used to convey vital messages, it is always better to accompany them with text. Concisely, subjects believe that the color has an ability to motivate, excite, draws attention to a particular matter and provides emphasis. If the color does not fulfil the intended purpose or deliver the right message, it can affect the credibility of the content and disturb greatly the aesthetic value of the interactive environment. Even though colour has an affective meaning, it should not be used alone. Most of Kansei generated by colours are universal, but some are influenced considerably by the culture. Therefore, when designing any computer interface, the designers should be careful enough when accommodating already defined general rules of color aesthetics. Thus Kansei Engineer can capture all these discrepancies since engineering procedure starts from the lowest level (basement of pyramid) and rise higher towards general Kansei. 


\section{Conclusion}

Kansei Engineering acquires the prospective users' feelings about the existing products and analyse them to localise or individualise it to users' preference needs. Since Kansei Engineering does not fully depend on already existing domain of specific values, it has a higher ability to engineer and incorporate the most user visual preferences into a particular design. Hence visual design is one of the important criteria that is used to distinguish a high quality products; it does not only satisfy users aesthetic needs, it also assists with improving the functionality of the interactive Interfaces; This research focuses on the affective quality of color to utilise it in Interactive Interface design. Understanding the affective quality is an extra aid to improve the simplicity, consistency, clarity and handling cultural differences. Good visual design is high quality, appropriate and relevant for the audience and the message that it is supporting. Quality of a product is not determined solely by its functional aspects, it also crucially depends on users' preferences, opinion and evaluation.

\section{References}

1. Nagamachi, M.: Workshop 2 on Kansei Engineering. In: Proceedings of International Conference on Affective Human Factors Design, Singapore (2001)

2. Lee, S., Harada, A., Stappers, P.J.: Pleasure with Products: Design based Kansei. In: Green, W., Jordan, P. (eds.) Pleasure with Products: Beyond usability, pp. 219-222. Taylor \& Francis, London (2002)

3. Osgood, C.E., Suci, G.J., Tannenbaum, P.H.: The Measurement of Meaning. University of Illions press, Urbana (1957)

4. Ou, L., Luo, M.R., Woodcock, A., Wright, A.: A study of colour emotion and colour preference, part 1: colour emotions for single colours. Color research and application 29, 232-240 (2004)

5. Xin, J.H., Cheng, K.M., Taylor, G., Sato, T., Hansuebsai, A.: Cross-regional comparison of colour emotions Part I: Quantitative Analysis. Color Research and Application 29, 451-457 (2004)

6. Tomomasa, N., Hidenori, T., Takashi, U.: Overview of Kansei engineering: a proposal of Kansei Informatics towards realising safety and pleasantness of individuals in information network society. The International Journal of Biometrics 1(1), 7 (2008) 